\title{
Artifacts Removal using Dragonfly Levenberg Marquardt-Based Learning Algorithm from Electroencephalogram Signal
}

\section{Quazi M. H}

Swami Ramanand Theerth Marathwada University

Nanded, Maharashtra, India

quazimateenh60@gmail.com

\author{
Dr. S. G. Kahalekar \\ Grahmin Polytechnic \\ Nanded, Maharashtra, India
}

\begin{abstract}
Electroencephalogram (EEG) is the recording of the electrical activity of the brain. The waveforms that are recorded from the brain regions show the cortical activity. The integration of EEG signals with other bio-signals is known as artifacts. Some of the artifacts are Electrooculogram (EOG), Electrocardiogram (ECG), and Electromyogram (EMG). The artifacts removed from the EEG signal are very challenging in medical. This paper presents the Dragonfly Levenberg Marquardt (DrLM) optimization-based Neural Network (NN) to remove the artifacts from EEG. Initially, the EEG signal is subjected to adaptive filter for determining the optimal weights based on Dragonfly Algorithm (DA) and LM. These two approaches are hybridized and given to the NN to identify the weights. At last, the artifacts are removed from the EEG signal. The performance of DrLM-NN is evaluated in terms of SNR, MSE, and RMSE. The proposed artifact removal method achieves the maximum SNR of 45.67, minimal MSE of 2982, and minimal RMSE of 1.11 that indicates its superiority.
\end{abstract}

Keywords: Electroencephalogram, Dragonfly optimization algorithm, Levenberg Marquardt algorithm, NARX model, Root mean square error

\section{Introduction}

EEG is one of the recordings of electrophysiological activity of the brain [1]. It is non-invasive in which the electrodes are placed across the scalp and is characterized by three components, such as frequency, amplitude, and shape. Based on these components, useful features or signatures in brain signals are extracted by various techniques. Here, the characteristics of the signals are differing from one to another, like normal or sleep/wakefulness. The brain waves are categorized based on their frequency ranges: beta 13-30 Hz, alpha 8-13 Hz, theta $4-8 \mathrm{~Hz}$, delta $0.5-4 \mathrm{~Hz}$, and gamma 30-128 Hz [1]. EEG is a significant tool for monitoring, managing, and diagnosing neurological disorders [2] [3]. Here, the EEG waves are contaminated by noises [7] [6]. The sources contain electrical noise at the range of 50 or $60 \mathrm{~Hz}$ [6]. Moreover, the utilities of EEG is less because of the artifacts produced by eye blinks, movement of the eye, line noise, the activity of the muscle, or noise from joint recordings. These noises are superposed with EEG data, effecting physical as well as statistical analysis of the brain signal [5].

Several methods are employed for the removal of EEG artifact from that two methods are most commonly used, namely Independent Component Analysis (ICA), and Adaptive Noise Cancellation (ANC) [10][9]. ICA is utilized for removing artifacts of muscular and ocular from the signal. In this framework, the data is collected from various channels, and the noise components are identified correctly [8] [7]. The signals are recorded using the variety of stimulus, like the movement of eyes, moving the limbs, and so on. The commonly utilized techniques for artifact removals are, such as NN [12] [9], adaptive filters, nonlinear PCA [13] [9], Principal Component Analysis (PCA) [11] [9], wavelet denoising, Autoregressive (AR) [14] [9]. The adaptive filters are utilized for removing the noise in real mode with limited computation complexity, but no assurance is given for the reference signal is the best signal. [7].

This paper presents DrLM optimization-based neural network for eliminating the artifacts from the EEG signal. Initially, the input signal is fed to adaptive filtering to remove the noise. Then, the optimal weights are determined based on the proposed dragonfly LM method. The proposed Dragonfly LM algorithm is the integration of DA and LM algorithm. Subsequently, the DA, as well as the LM algorithm [23], are hybridized and fed to the NN to determine the optimal weights. After that, the developed filtering method performs the removal of the artifacts from the EEG signal. 
The main contribution of the research paper is developing artifact removal approach using the proposed DrLM-NN for removing the artifacts effectively based on the optimal weights.

The organization of the paper is as follows: Section 2 illustrates the literature survey, along with the challenges of artifacts removal. Proposed DrLM-NN is described in section 3 deals. Section 4 provides the results and discussions, and finally, concludes the paper in section 5 .

\section{Literature Survey}

Various literature surveys are reviewed in this section. M. Chavez et al.[1] developed Surrogate-Based Artifact Removal (SuBAR) for removing muscular, and ocular noises from EEG. This framework integrated resampling method, and the wavelet decomposition termed as surrogate data. Based on timefrequency analysis, this method was capable to find, and automatically filter ocular as well as muscular noises in single-channel EEG. The method failed to consider other optimizations algorithms for better performance. Ben Somers et al.[2] designed the EEG artifact removal tool to eliminate the artifacts from EEG. This tool was based on Multi-channel Wiener filtering (MWF). Here, the artifact covariance matrix was replaced using generalized eigen value decomposition. This approach was validated using real data, and hybrid EEG data. The method did not consider temporal artifact detection approaches for removing the artifacts automatically based on signal statistics. Aysa Jafarifarmand, and Mohammad Ali Badamchizadeh [3] developed adaptive FLN-RBFN-based filter for removing muscular, ocular, and cardiac noises from EEG signals. Here, the adaptive NN was not able to determine the weights, when the learning process was done. Therefore, adaptive filtering was utilized for finding the optimal weights, and to obtain a good system performance. M.H. Quazi, and S.G. Kahalekar [4] developed Firefly and Levenberg Marquardt (FLM) -based learning approach for eliminating the artifacts from EEG signal. Initially, the input signal was subjected to the adaptive filter to yield the optimal weights based on FLM. These two approaches were hybridized effectively and given to the NN. After that, the filtering was employed for removing artifacts effectively. The method failed to consider optimization algorithms.

\subsection{Challenges}

This section deals with the challenges faced by the existing techniques.

- Several Artifact Removal Techniques (ARTs) are focussed to handle few single types or specific types of EEG artifacts, but the removal of multiple types of artifacts is still challenging [15].

- Another challenge faced by EEG is having very small Signal-to-Noise Ratio (SNR) of brain waves, due to the coupling of various noise sources, failed to observe [24]. Also, the brain signals have limited amplitudes hence it is simply contaminated by noise.

- The major disadvantages of EEG artifact removal issue are the selection of threshold value. The main reason behind this is the raw EEG signal coefficients are not removed and also not keep the noise signals as the raw ones [6].

\section{Proposed Artifact Removal using Dragonfly Levenberg Marquardt Optimization-Based Learning Algorithm}

This section presents the proposed artifact removal method using DrLM-NN. At first, the input signal is given to the filtering. The filtering is performed using an adaptive filter to eliminate the artifacts from the EEG signal [13]. After filtering, the multi-channel artifact removal is carried out using the proposed optimization algorithm, termed DrLM. The DrLM is developed by integrating DA and LM. Then, the integrated algorithms are hybridized and fed to the $\mathrm{NN}$ for the determination of weights for adaptive filtering. Fig. 1 depicts the schematic diagram of the proposed artifact removal method. 
EEG

Signal

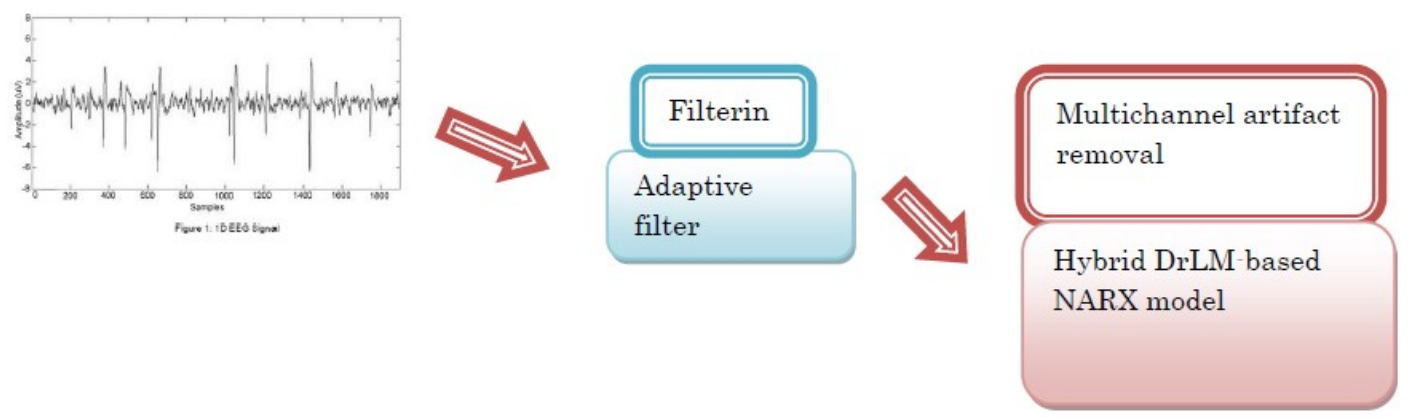

Fig. 1. Schematic diagram of the proposed artifact removal method

\subsection{Removal of Artifacts Using Adaptive Filtering}

Adaptive noise cancelation is utilized for removing the artifacts from brain waves. This cancelation process consists of two inputs. Here, the first one is taken from the EEG signal and the second input is collected from the noise signal. After that, the artifacts signal is subjected to the unidentified non-linear dynamics to generate the interference signal. After the generation of the noise signal, the perfect signal $\mathrm{P}(s)$, and the interference signal are combined to form the input signal and it is expressed as,

$$
\mathrm{P}(\mathrm{s})=\mathrm{D}(\mathrm{s})+\mathrm{J}(\mathrm{s})
$$

where, $\mathrm{D}(\mathrm{s})$ represents the input signal taken from the EEG, and the $\mathrm{J}(\mathrm{s})$ represents the noise signal that is obtained from the noise source. The adaptive noise cancellation signal is represented as,

$$
\mathrm{D}_{\mathrm{o}}(\mathrm{s})=\mathrm{D}(\mathrm{s})+\mathrm{J}(\mathrm{s}) \quad \mathrm{E}(\mathrm{s})
$$

where, E(s) signifies the filtered output.

\subsection{Nonlinear Autoregressive with Exogenous (NARX) Neural Network model}

This section explains the NARX NN [16] model for modeling the nonlinear systems. The NARX is defined by combining a recurrent loop, time delay, and multilayer feedforward network. The NARX system model consists of three layers, such as output, input, and hidden layers. The input layer comprises of delayed regressed output vector, delayed exogenous input vector, and the exogenous input vector. Once the NN operation is done, the output vector is represented as $\mathrm{K}(\mathrm{m}+1)$.

The mathematical expression for the NARX neural network model is expressed as,

$$
\mathrm{K}(\mathrm{m}+1)=\mathrm{f}\left(\mathrm{K}(\mathrm{m}), \ldots,\left(\mathrm{K}\left(\mathrm{m}-\mathrm{Q}_{\mathrm{K}}\right) ; \mathrm{U}(\mathrm{m}), \ldots, \mathrm{U}\left(\mathrm{m}-\mathrm{Q}_{\mathrm{U}}\right)\right.\right.
$$

where, $K(m)$ refer to the exogenous input vector, and the delayed regressed output vector is denoted as $\mathrm{K}(\mathrm{m}-1), \mathrm{K}(\mathrm{m}-2), \ldots,\left(\mathrm{K}\left(\mathrm{m}-\mathrm{Q}_{\mathrm{K}}\right)\right.$, and the delayed exogenous input vector is represented as, $\mathrm{U}(\mathrm{m}), \mathrm{U}(\mathrm{m}-1), \ldots, \mathrm{U}\left(\mathrm{m}-\mathrm{Q}_{\mathrm{U}}\right)$. In the primary stage, the weights are assigned to the hidden units, and the exogenous input vector, and between the regressed output vector and hidden units.

\subsection{Artifact Removal Based on Proposed Dragonfly-LM Optimization Algorithm}

Dragonfly Algorithm (DA) [17] is adopted to compute the optimal weights, which are determined by an error function. DA is the meta-heuristic algorithm to solve discrete, single-objective as well as multiobjective problems. DA has competitive performance in terms of local optima avoidance, convergence behavior, and so on, with other meta-heuristic methods. The weights obtained from both the algorithms are integrated using hybrid learning, and new weight vectors are obtained.

$$
\varpi^{\mathrm{bc}}=\varpi^{\mathrm{d} 1}+\varpi^{\mathrm{d} 2}+\ldots+\varpi^{\mathrm{dl}}
$$

The algorithmic steps of the DF algorithm are as follows,

a) Initialization: In the initial step, the population of the dragon flies is randomly initialized. Let us assume the size of the DA is L. The values of the solution encoding vectors synchronize with the population of the DA is expressed as,

$$
\mathrm{L}=\left\{\mathrm{L}_{1}, \mathrm{~L}_{2}, \ldots \ldots, \mathrm{L}_{\mathrm{K}}\right\}
$$


b) Changing the Location of Record: The location of the records is changed based on the cluster size and generalization level. The location update is given as,

$$
\begin{aligned}
& \mathrm{L}_{\mathrm{x}}^{*}=\mathrm{L}_{\mathrm{x}}(\tau+1) \\
& \mathrm{L}_{\mathrm{x}}(\tau+1)=\mathrm{L}_{\mathrm{x}}(\mathrm{t} \tau)+\Delta \mathrm{L}_{\mathrm{x}}(\tau+1)
\end{aligned}
$$

where, $\Delta \mathrm{L}_{\mathrm{x}}(\tau+1)$ denotes the changing the position of the records, and is given by,

$$
\Delta \mathrm{L}_{\mathrm{x}}(\tau+1)=\left(\mathrm{fN}_{\mathrm{x}}+\mathrm{cO}_{\mathrm{x}}+\mathrm{bP}_{\mathrm{x}}+\mathrm{aQ}_{\mathrm{x}}+\mathrm{dR}_{\mathrm{x}}\right)+\mathrm{jL}_{\mathrm{x}}(\tau)
$$

where, $P_{x}$ signifies the cohesion of records, $O_{x}$ refer to the alignment, $Q_{x}$ represents the attraction due to a food source $X_{g}, R_{x}$ indicates the position of enemy records, and $N_{x}$ denotes the separation of records. The neighboring record $\mathrm{L}_{\mathrm{uv}}$ and the location of record $\mathrm{L}_{\mathrm{x}}$ gets changed for each factor of separation, alignment, cohesion, attraction, and the position of the enemy are expressed below,

$$
\begin{gathered}
\mathrm{N}_{\mathrm{x}}=\sum_{\mathrm{u}=1}^{\mathrm{K}}\left(\begin{array}{ll}
\mathrm{L}_{\mathrm{x}} & \mathrm{L}_{\mathrm{u}}
\end{array}\right) \\
\mathrm{O}_{\mathrm{z}}=\frac{\sum_{\mathrm{u}=1}^{\mathrm{K}} \mathrm{S}_{\mathrm{u}}}{\mathrm{K}} \\
\mathrm{O}_{\mathrm{x}}=\frac{\sum_{\mathrm{u}=1}^{\mathrm{K}} \mathrm{L}_{\mathrm{u}}}{\mathrm{K}} \mathrm{L}_{\mathrm{x}} \\
\mathrm{Q}_{\mathrm{x}}=\left(\begin{array}{ll}
\mathrm{L}^{\alpha} & \mathrm{L}_{\mathrm{x}}
\end{array}\right) \\
\mathrm{R}_{\mathrm{x}}=\left(\begin{array}{ll}
\mathrm{L}^{\beta} & \mathrm{L}_{\mathrm{x}}
\end{array}\right)
\end{gathered}
$$

c) Evaluation of fitness: The fitness function is computed for the individual solution using the Eq. (14) for better result. The output having minimum fitness value is considered as the k-anonymization database. The $\mathrm{k}$-anonymization database is a property possessed by certain anonymized data.

$$
\mathrm{L}_{\mathrm{x}}^{*}=\operatorname{Min}\left(\operatorname{Fitness}\left(\mathrm{L}_{\mathrm{x}}^{*}(\tau)\right)\right)
$$

d) Termination: After a certain iteration limit, the algorithm terminates, and the optimal solution is retained at the end of the procedure.

\subsection{DrLm Based NARX for Multi-Channel Artifacts Removal}

Once the hybridization is performed, the artifacts are removed using DrLM-based NARX model. The brain activity is measured using the electroencephalogram approach. Here, 15 electrodes are used and are placed on several regions of the scalp, which is known as the multi-channel signal. Subsequently, the signals produced from various electrodes are subjected to the NARX model based on the proposed approach. Thus, the NARX model produced a clean EEG signal for the corresponding electrodes, without making any noise signal, like EOG, ECG, and EMG signal.

\section{Discussion of Results}

This section describes the results and discussion made by the proposed approach for eliminating the artifacts from the EEG signal.

\subsection{Experimental Setup}

The experimentation of the proposed technique of artifact removal is performed in the system with 2 GB RAM, Intel i-3 core processor, Windows 10 Operating System. The proposed method is executed in MATLAB.

\subsection{Database Description}

The input signal is taken from PhysioNet database [19], which is the real-time signal contaminated with several noise sources like, Electro-Oculogram (EOG) signal [21], Electromyogram (EMG) signal [20], and Electrocardiogram (ECG) signal [22]. The input database chosen for EEG is CHB-MIT scalp EEG database [22]. 


\subsection{Performance Metrics}

The evaluation of the proposed technique is performed using three metrics, namely Signal to Noise Ratio (SNR), Mean Square Error (MSE), and Root Mean Square Error (RMSE).

The SNR is a measurement for comparing the similarity between the watermarked host signal and the undisturbed host signal.

$$
\mathrm{SNR}=10 \log _{10} \frac{\sum_{\mathrm{j}=1}^{\mathrm{m}}\left(\mathrm{U} \quad \mathrm{U}^{\prime}\right)^{2}}{\sum_{\mathrm{j}=1}^{\mathrm{m}}(\mathrm{U})^{2}} \mathrm{~dB}
$$

The RMSE of the methods is given as,

$$
\operatorname{RMSE}_{j}={\left.\sqrt{\frac{1}{\mathrm{v}} \sum_{\mathrm{j}=1}^{\mathrm{v}}\left(\mathrm{y}_{\mathrm{ij}} \hat{\mathrm{y}}_{\mathrm{ij}}\right.}\right)^{2}}^{2}
$$

Where, v refers to the total pixel, $y_{\mathrm{ij}}$ signifies the real abundance fraction for $\mathrm{i}^{\text {th }}$ end member in $\mathrm{j}^{\text {th }}$ pixel, and $\hat{y}_{\mathrm{ij}}$ refers to the corresponding estimated value. Thus,

$$
\mathrm{RMSE}=\sum_{\mathrm{j}=1}^{\mathrm{q}} \mathrm{RMSE}_{\mathrm{j}}
$$

Where, q symbolizes the extracted end members and $\mathrm{RMSE}_{\mathrm{j}}$ corresponds to the RMSE of the individual pixels.

\subsection{Comparative Analysis}

The comparative analysis of the proposed DrLM-NN by evaluating the performance of other comparative techniques is elaborated in this section. The comparative analysis is performed by varying the signal, and the results are evaluated based on SNR, MSE, and RMSE.

\subsection{Competing methods}

The methods, like NN, ICA, WICA, FICA, cascaded adaptive filter, and NN-PROP, are utilized for the comparison with the proposed DrLM-NN for the analysis.

\subsubsection{Comparative analysis of EEG signal with ECG artifact}

The comparative analysis of the proposed DrLM-NN method is analyzed in terms of SNR, MSE, and RMSE with ECG artifact is depicted in Fig. 2. Fig. 2a) shows the analysis based on SNR with different signals. For signal 1, the existing techniques, like WICA, NN-FLM, ICA, NN-LM, cascaded adaptive filter, and, FICA, possesses the SNR of 7.1, 43.17, 5.82, 43.19, 38.35, and 10.14, respectively, which is comparatively lower than the proposed method. For the same signal, the DrLM-NN acquired the SNR of 45.67. Similarly, for signal 5, the methods, NN-LM, FICA, ICA, cascaded adaptive filter, WICA, and NNFLM attained the SNR of 43.4, 5.1, 3.45, 38.1, 3.32, and 43.52, respectively, whereas the SNR of the DrLM-NN is 44.4. From the above interpretation, it is seen that the DrLM-NN achieved improved SNR of 45.67 .

The analysis in terms of MSE is depicted in Fig. 2b). Here, for signal 2, the existing techniques, like WICA, NN-FLM, ICA, NN-LM, cascaded adaptive filter, and FICA achieved the MSE of 7842.5, 4210, 7852.1, 4312, 5200, and 7854.4, respectively, but the DrLM-NN acquired the MSE of 3819.8. Similarly, for signal 4, the existing techniques, such as ICA, WICA, FICA, NN-LM, NN-FLM, and cascaded adaptive filter achieved the MSE of 5298, 5372, 5312, 4118, 4102, and 4521, respectively. Meanwhile, the DrLM-NN attained the MSE of 2982.

The comparative analysis in terms of RMSE is depicted in Fig. 2c). For signal 3, the existing methods, like NN-FLM, ICA, NN-LM, FICA, WICA, and cascaded adaptive filter acquires the RMSE of 2, 86, 2, 84, 88, and 21, respectively. Meanwhile, the DrLM-NN obtained the RMSE value of 1.312. Similarly, for signal 5, the RMSE value of the existing methods, like a cascaded adaptive filter, ICA, NNFLM, WICA, NN-LM, and FICA is 20, 89, 2.2, 87, 2.2, and 86, respectively, whereas the DrLM-NN attained the RMSE of 1.23. 


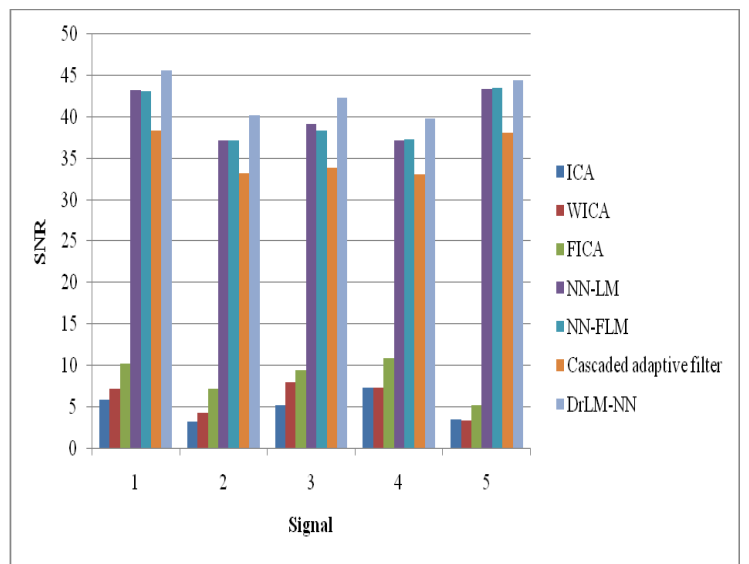

a)

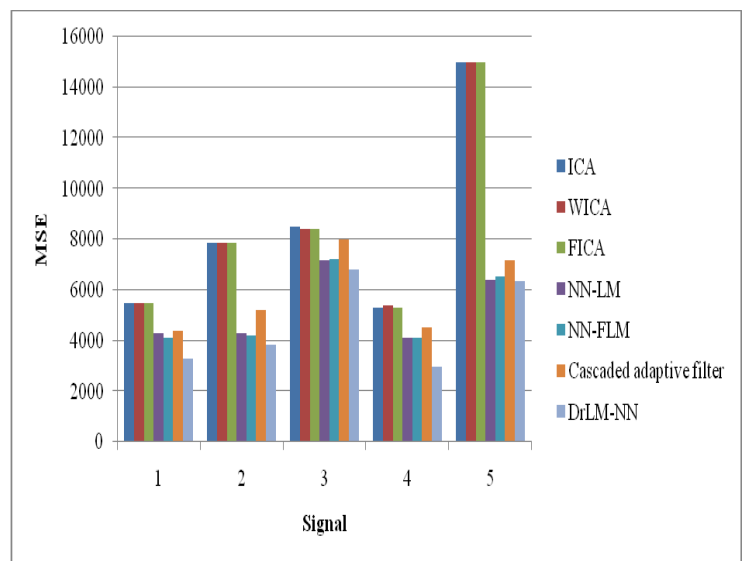

b)

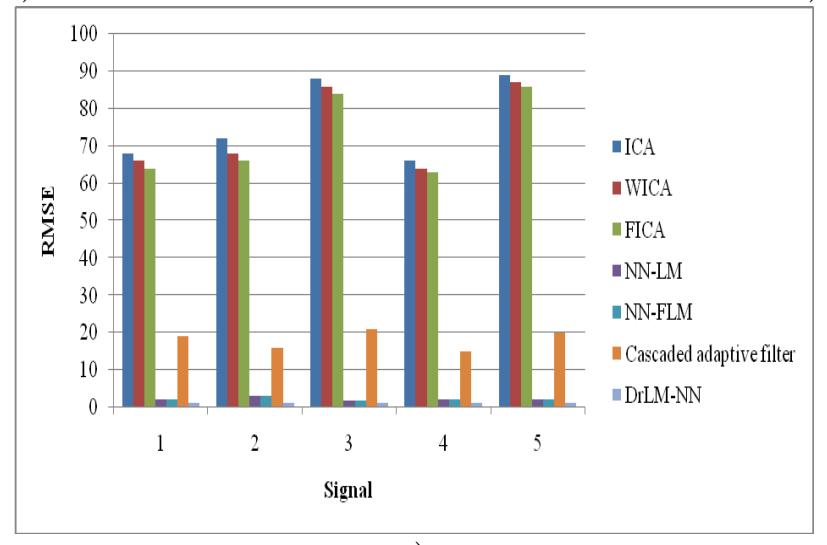

c)

Fig. 2 Comparative analysis by varying the signal with ECG artifact (a) SNR, (b) MSE (c) $R M S E$

\subsubsection{Comparative analysis of EEG signal with EMG artifact}

Fig. 3 shows the comparative analysis of the proposed method in terms of SNR, MSE, and RMSE with ECG artifact. Fig. 3a) shows the analysis based on SNR with different signals. For signal 1, the existing techniques, like WICA, ICA, NN-LM, FICA, NN-FLM, and cascaded adaptive filter, possesses the SNR of $6.92,8.23,6.72,42.93,41.32$, and 39.2, respectively, which is comparatively lower than the proposed method. For the same signal, the DrLM-NN acquired SNR of 44.34. Similarly, for signal 5, the methods, WICA, NN-LM, NN-FLM, FICA, cascaded adaptive filter, and ICA, attained SNR of 3.42, 43.42, 44.2, 3.15, 39.5, and 3.12, respectively, whereas the SNR of the DrLM-NN is 45.21. From the above interpretation, it is seen that the DrLM-NN achieved improved SNR of 45.21.

The analysis in terms of MSE is depicted in Fig. 3b). Here, for the signal 2, the existing techniques, like ICA, FICA, NN-LM, WICA, NN-FLM, and cascaded adaptive filter achieved MSE of 6212, 6314, 5112, 6234, 4122, and 4532, respectively, but the DrLM-NN acquired MSE of 3146. Similarly, for signal 4, the existing techniques, such as WICA, ICA, FICA, NN-FLM, NN-LM, and cascaded adaptive filter achieved MSE of 5622, 5600, 5618, 4310, 4321, and 4500, respectively. Meanwhile, the DrLM-NN attained the MSE of 4150.

The comparative analysis in terms of RMSE is depicted in Fig. 3c). For signal 3, the existing methods, like ICA, FICA, WICA, NN-FLM, NN-LM, and cascaded adaptive filter acquires the RMSE of 90, 82, 84, 1.51, 1.51, and 19, respectively. Meanwhile, the DrLM-NN obtained the RMSE value of 1.52. Similarly, for signal 5, the RMSE value of the existing methods, like ICA, WICA, FICA, NN-LM, NNFLM, and the cascaded adaptive filter is $85,84,83,1.21,1.21$, and 20 , respectively, whereas the DrLMNN attained the RMSE of 1.11 . 


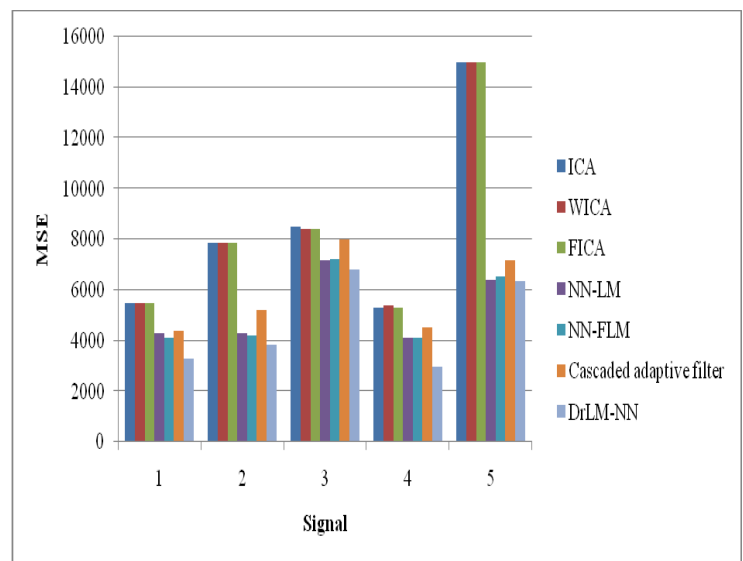

a)

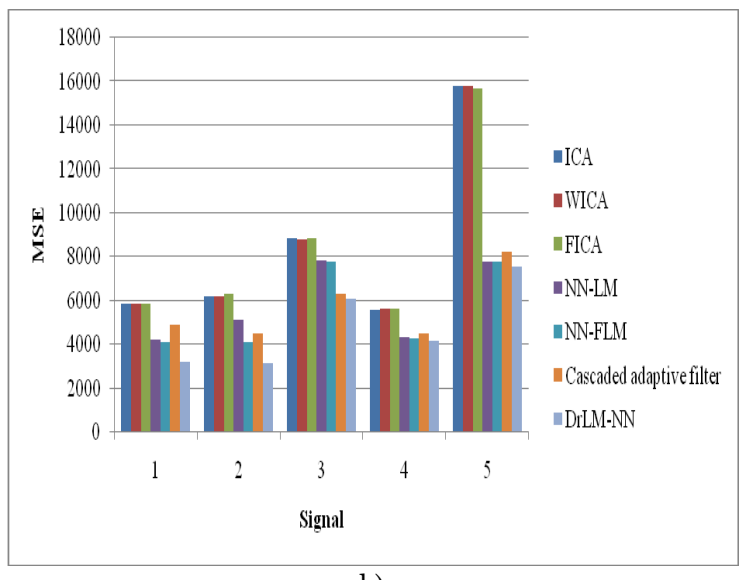

b)

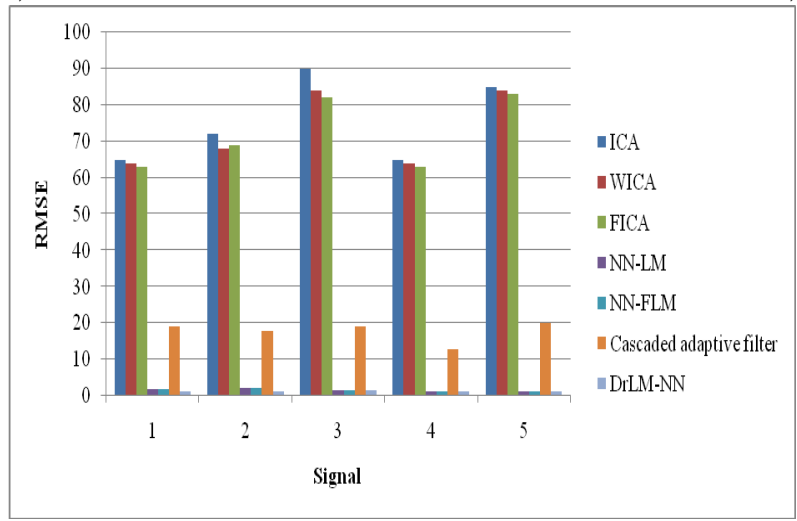

c)

Fig. 3 Comparative analysis by varying the signal with EMG artifact (a) SNR, (b) MSE (c) RMSE

\subsubsection{Comparative analysis of EEG signal with EOG artifact}

The comparative analysis of the proposed DrLM-NN method is analyzed based on SNR, MSE, and RMSE with ECG artifact is illustrated in Fig. 4. The analysis based on SNR with different signals is depicted in Fig. 4a). For signal 1, the existing techniques, such as WICA, FICA, ICA, NN-LM, NN-FLM, and cascaded adaptive filter, possesses SNR of 5.3, 7.2, 66.72, 43.2, 43.12, and, 39.5, respectively, which is comparatively lower than the proposed method. For the same signal, the DrLM-NN acquired the SNR of 45.6. Similarly, for signal 5, the methods, ICA, WICA, FICA, NN-LM, NN-FLM, and cascaded adaptive filter, attained the SNR of 2.12, 3.12, 3.45, 42.1, 42.34, and 38.1, respectively, whereas the SNR of the DrLM-NN is 39.3. From the above interpretation, it is seen that the DrLM-NN achieved improved SNR of 45.6. The analysis in terms of MSE is depicted in Fig. 4b). Here, for the signal 2, the existing techniques, like ICA, WICA, FICA, NN-LM, NN-FLM, and cascaded adaptive filter achieved the MSE of 6531, 6521, 6540, 5721, 4341, and 5121, respectively, but the DrLM-NN acquired the MSE of 3010. Similarly, for signal 4, the existing techniques, such as ICA, WICA, FICA, NN-LM, NN-FLM, and cascaded adaptive filter achieved the MSE of 5321, 5318, 5317, 4212, 4110, and 4312, respectively. Meanwhile, the DrLM-NN attained the MSE of 3090. The comparative analysis in terms of RMSE is depicted in Fig. 4c). For signal 3, the existing methods, like ICA, WICA, FICA, NN-LM, NN-FLM, and cascaded adaptive filter acquires the RMSE of $87,86,83,1.09,1.09$, and 20 , respectively. Meanwhile, the DrLM-NN obtained the RMSE value of 1.112. Similarly, for signal 5, the RMSE value of the existing methods, like ICA, WICA, FICA, NN-LM, NN-FLM, and the cascaded adaptive filter is $85,84,83,1.32$, 1.32, and 22, respectively, whereas the DrLM-NN attained the RMSE of 1.321. 


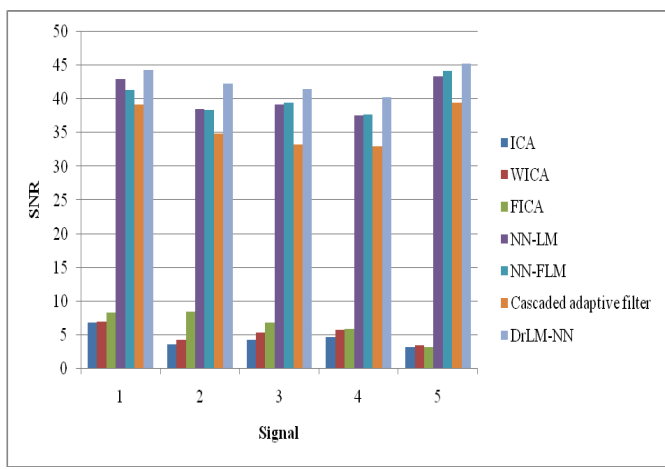

a)

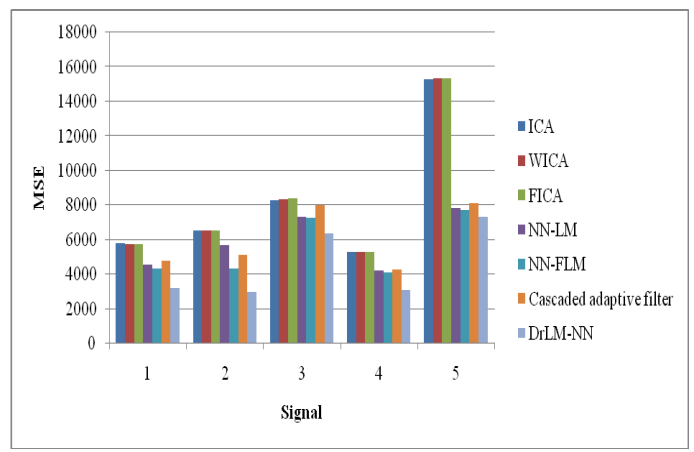

b)

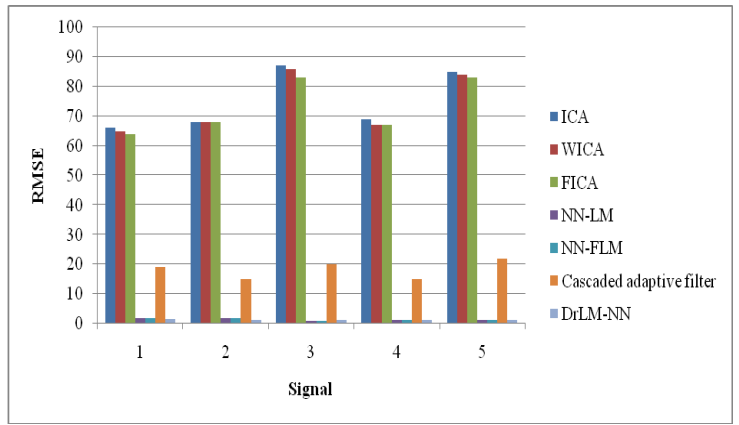

c)

Fig. 4 Comparative analysis by varying the signal with EOG artifact (a) SNR, (b) MSE (c) RMSE

\subsection{Comparative Discussion}

Table 1 describes the discussion to reveal the best performance attained by the artifact methods, based on SNR, MSE, and RMSE. ICA acquired the SNR, MSE, and RMSE of 7.34, 5298, and 65, respectively, while WICA attains the SNR, MSE, and RMSE of 7.9, 5372, and 64. The SNR, MSE, and RMSE values of FICA are 10.91, 5312, and 63, respectively, and also NN-LM attains the SNR, MSE, and RMSE of 43.4, 4118, AND 1.21. The NN-FLM has the SNR, MSE, and RMSE of 43.52, 4100.1, and 1.21, and the SRN, MSE, and RMSE values of cascaded adaptive filter are 38.35, 4392, and 13, respectively. Among all the comparative methods, DrLM-NN possesses improved performance with SNR, MSE, and RMSE, of $95.90 \%, 45.67,2982$, and 1.11, respectively. Here, the bold value indicates the superior performance of the both the proposed and conventional methods.

Table 1 Comparative discussion

\begin{tabular}{l|l|l|l}
\hline Methods & SNR & MSE & RMSE \\
\hline ICA & 7.34 & 5298 & 65 \\
WICA & 7.9 & 5372 & 64 \\
FICA & 10.91 & 5312 & 63 \\
NN-LM & 43.4 & 4118 & $\mathbf{1 . 2 1}$ \\
NN-FLM & 43.52 & 4100.1 & $\mathbf{1 . 2 1}$ \\
Cascaded adaptive filter & 38.35 & 4392 & $\mathbf{1 3}$ \\
Proposed DrLM-NN & $\mathbf{4 5 . 6 7}$ & $\mathbf{2 9 8 2}$ & $\mathbf{1 . 1 1}$ \\
\hline
\end{tabular}

\subsection{Conclusion}

This paper presents the hybrid optimization approach for the NN-enhanced adaptive filtering for removing the artifacts from multi-channel EEG data. The proposed DrLM-NN method is employed for determining the weights of NARX neural network, which is designed by combining the LM and FF algorithm to solve individual shortcomings of the two approaches. Experimentation is carried out using physionet database. Here, 15 channels are considered and the artifact signals, like EOG, EMG, and ECG are included for the analysis and is implemented using MATLAB. The performance of the DrLM-NN is evaluated using SNR, MSE, and RMSE. The proposed method produces the maximal SNR 45.67of 96.4\%, the minimum MSE of 2982, and the minimal RMSE of 1.11, that indicates the superiority of the proposed method. The future dimension of the research will be concentrated on extending the analysis using other standard databases with highly advanced features. 


\section{Compliance with Ethical Standards}

Conflicts of interest: Authors declared that they have no conflict of interest.

Human participants: The conducted research follows the ethical standards and the authors ensured that they have not conducted any studies with human participants or animals.

\section{References}

[1] M. Chavez, F. Grosselin, A. Bussalb, F. De Vico Fallani, and X. Navarro-Sune, "Surrogate-based artifact removal from single-channel EEG", IEEE transactions on neural systems and rehabilitation engineering, 2017.

[2] Ben Somers, Tom Francart, and Alexander Bertrand,"A generic EEG artifact removal algorithm based on the multi-channel Wiener filter", vol.15, no.3, pp.036007, 2018.

[3] Aysa Jafarifarmand, and Mohammad Ali Badamchizadeh, "Artifacts removal in EEG signal using a new neural network enhanced adaptive filter", Neurocomputing, vol.103, pp. 222-231, 2013.

[4] M.H. Quazi, and S.G. Kahalekar, "Artifacts removal from EEG signal: FLM optimization-based learning algorithm for neural network-enhanced adaptive filtering", Bio-cybernetics, and biomedical engineering, 2017.

[5] Jidong Hou, Kyle Morgan, Don M. Tucker, Amy Konyn, Catherine Poulsen, Yasuhiro Tanaka, Erik W. Anderson, and Phan Luu, "An improved artifacts removal method for high dimensional EEG", Journal of neuroscience methods, vol.268, pp.31-42, 2016.

[6] M.M. Hartmann, K.Schindler, T.A.Gebbink, G.Gritsch, and T.Kluge, "PureEEG: Automatic EEG artefact removal for epilepsy monitoring", Neurophysiologie Clinique/Clinical Neurophysiology, vol.44, no.5, pp.479-490, 2014.

[7] William O. Tatum, Barbara A. Dworetzky, and Donald L. Schomer, "Artifact and recording concepts in EEG", vol. 28, no.3, pp.252-63, 2011.

[8] Tzyy-Ping Jung, Scott Makeig, Colin Humphries, Te-Won Lee, Martin J. Mckeown, Vicente Iragui, and Terrence J. Sejnowski,"Removing electroencephalographic artifacts by blind source separation",Psychophysiology, vol.37, pp.163-178, 2000.

[9] Arjon Turnip, and Edy Junaidi, "Removal Artifacts from EEG Signal Using Independent Component Analysis and Principal Component Analysis", In proceedings of second international conference on Technology, Informatics, Management, Engineering and Environment, August 2014.

[10] G. Geetha, and S. N. Geethalakshmi, "Artifact Removal from EEG using Spatially Constrained Independent Component Analysis and Wavelet Denoising with Otsu's Thresholding Technique," Procedia Engineering, vol. 30, pp. $1064-1071,2012$.

[11] T. Liu, and D. Yao, "Removal of the ocular artifacts from EEG data using a cascaded spatio-temporal processing," Computer methods and programs in biomedicine, vol. 83, pp. 95-103, 2006.

[12] H.-A. T. Nguyen, J. Musson, F. Li, W. Wang, G. Zhang, R. Xu, C. Richey, T. Schnell, F. D. McKenzie, and J. Li,"EOG artifact removal using a wavelet neural network," Neurocomputing, vol. 97, pp. 374-389, 2012.

[13] A. Turnip, D. Soetraprawata, and D. E. Kusumandari, "A Comparison of EEG Processing Methods to Improve the Performance of BCI," International Journal of Signal Processing Systems, vol. 1, no. 1, pp. 63-67, 2013.

[14] A. Turnip and K.-S. Hong, "Classifying Mental Activities From EEG-P300 Signals Using Adaptive Neural Networks," International Journal of Innovative Computing, Information and Control, vol. 8, no. 9, pp. 64296443, 2012.

[15] Sim Kuan Goh, Hussein A. Abbass, Kay Chen Tan, Abdullah Al-Mamun, Chuanchu Wang, and Cuntai Guan,"Automatic EEG Artifact Removal Techniques by Detecting Influential Independent Components", IEEE transactions on emerging topics in computational intelligence, vol. 1, no. 4, August 2017.

[16] Jose Maria P. Junior and Guilherme A. Barreto, "Long-Term Time Series Prediction with the NARX Network: An Empirical Evaluation", March 2007.

[17] Seyedali Mirjalili,"Dragonfly algorithm: a new meta-heuristic optimization technique for solving single-objective, discrete, and multi-objective problems", Neural computing and applications, May 2015.

[18] Vilas H. Gaidhane, Vijander Singh, Yogesh V. Hote, Mahendra Kumar,"New Approaches for Image Compression Using Neural Network", Journal of Intelligent Learning Systems and Applications, vol.3, pp.220-229, 2011.

[19] PhysioNet dataset taken from" (https://physionet.org/cgi-bin/atm/ATM?", accessed on May 2019.

[20] EMG signal is taken from,"(https://physionet. org/physiobank/database/emgdb/emg_healthy.txt), accessed on My 2019.

[21] EOG signals is taken from physionet," (https://physionet.org/cgi-bin/atm/ATM?database= ptbdb\&tool=plot_waveforms), accessed on May 2019.

[22] CHB-MIT scalp EEG database is taken from (https://datamed.org/displayitem.php?repository $=0021 \& i d=590$ ce9f05152c6571c0cd5ad\&query=).

[23] C. Peng and L. Yanbing, "Model in Frequency-Domain Identification of a Fast Steering Mirror System Based on Levenberg-Marquardt Algorithm," 2017 2nd International Conference on Cybernetics, Robotics and Control (CRC), Chengdu, 2017, pp. 199-202. 